\title{
Isolation of 2,4-Di-tert-butylphenol and Butyrospermol 3- $\beta$-O-palmitate from Syzygium aqueum stem bark
}

\begin{abstract}
Syzygium genus in Myrtaceae family is a rich source of phytochemical constituents that possess various bioactivities. One of well-known species of this genus, Syzygium aqueum, has been already utilized as traditional medicine. This plant is cultivated in countries of tropical regions of the world such as Malaysia and Indonesia. The aim of this study was intended to isolate phytochemical constituents from the stem bark of $S$. aqueum. To date, there have been no reports of chemical substances isolated from the stem bark of this plant. The powder of the stem bark was extracted followed by partitioned in order to obtain $\mathrm{n}$-hexane extract which then was separated using silica gel column chromatographic several times until the pure compounds were resulted. The isolated compounds were identified by spectroscopie method including Fouirer Transform Infrared (FTIR) and nuclear magnetic resonance (NMR) and were known as 2,4-Di-tert-butylphenol \& butyrospermol 3- $\beta$ - $O$ palmitate, respectively. The spectoscopie data of those compounds were compared with references.
\end{abstract}

Keywords: Myrtaceae, Syzygium aqueum, phytochemical constituents, phenolic, ester of fatty acid, 2,4-di-tert-butylphenol and butyrospermol 3- $\beta$ - $O$-palmitate
Volume 5 Issue 4 - 2020

\author{
Ei Ei Aung, ${ }^{1,2}$ Alfinda Novi Kristanti, ${ }^{3,4}$ \\ Nanik Siti Aminah, ${ }^{3,4}$ Yoshiaki Takaya, ${ }^{5}$ Rico \\ Ramadhan ${ }^{3}$ \\ 'PhD Student of Mathematics and Natural Sciences, Faculty of \\ Science and Technology, Universitas Airlangga, Indonesia \\ ${ }^{2}$ Department of Chemistry, Yadanarbon University, \\ Amarapura-05063, Mandalay, Myanmar \\ ${ }^{3}$ Department of Chemistry, Faculty of Science and Technology, \\ Universitas Airlangga, Indonesia \\ ${ }^{4}$ Biotechnology of Tropical Medicinal Plants Research Group, \\ Universitas Airlangga, Surabaya, Indonesia \\ ${ }^{5}$ Faculty of Pharmacy, Meijo University, Tempaku-468-8502, \\ Nagoya, Japan, Department of Chemistry, University of Mandalay, \\ Myanmar
}

\begin{abstract}
Correspondence: Alfinda Novi Kristanti, Department of Chemistry, Faculty of Science and Technology, Universitas Airlangga, Komplek Kampus C Unair, Jl. Mulyorejo-60II5, Surabaya, Indonesia, Tel +62-31-5936501, Fax +62-3I-5936502, Email alfinda-n-k@fst.unair.ac.id
\end{abstract}

Received: August 14, 2020 | Published: August 3I, 2020

\section{Introduction}

Myrtaceae is an enormous family of wood flowering plants with 155 genera and around about 4000 species. The species are cultivated in South America, Southeast Asia, and Australia, but few species are found in Africa. ${ }^{1,2}$ Tropical regions, such as Malaysia and Indonesia, are native regions for one species of this family which is Syzygium aqueum. ${ }^{3,4} S$. aqueum has the local name water jambu or water apple. The whole parts of the tree have been applied as traditional medicine such as for relieving child birth pains (bark), treating a cracked tongue (leaves), relieving itching and reducing swelling (roots) $)^{5-7}$ Moreover, the previous researchers have been tested leaves and fruits for antioxidant, anti-inflammatory, and anticancer (MCF-7 and MDA-MB-231 cell lines) activities. ${ }^{8-11}$ In recent, there are some reports about the phytochemicals constituents isolated from leaves such as flavonoid and tannin and some of these flavonoids have been determined the antidiabetic and antioxidant activities. ${ }^{1,6,12}$ The phytochemical compounds of stem bark of $S$. aqueum have never been reported by any researchers. Therefore, the present study was carried out to collect the phytochemicals compounds isolated from stem bark of this plant.

\section{Material and method}

\section{General experimental procedure}

FT-IR spectra (Fouirer Transform Infrared spectroscopy) were determined on Tracer-100 spectrophotometer (Shimadzu) and the spectra showed in $\mathrm{cm}^{-1} .{ }^{1} \mathrm{H} \quad \mathrm{NMR},{ }^{13} \mathrm{C}$ NMR (Nuclear Magnetic Resonance) and 2D NMR (HSQC, HMBC and COSY) were recorded on BRUKER $600 \mathrm{~Hz}$ using Tetramethylsilane (TMS). Chemical shifts of carbon and proton NMR were given in $\delta$ (ppm).

\section{Plant material}

The stem bark of $S$. aquem was collected form Wage, Taman, Sidoarjo, East Java, Indonesia. This our sample had brown color and cracked. Department of Biology, Faculty of Science and Technology, Universitas Airlangga was identified and determined the scientific name. The stem bark obtained was crushed to form a powder and then was extracted using a general protocol. ${ }^{13}$

\section{Extraction and isolation}

The powdered of stem bark of $S$. aqueum $(1 \mathrm{~kg})$ was macerated with $40 \mathrm{~L}$ of methanol at room temperature for 3 days. The methanol extract $(450 \mathrm{~g})$ was partitioned with $\mathrm{n}$-hexane. n-hexane fraction obtained $(50 \mathrm{~g})$ was then separated using silica gel 60 (700-200 mesh ASTM) in gravity column chromatographic method (GCC) with n-hexane: ethyl acetate (9:1) as eluent. ${ }^{13}$ The similar fractions were combined to yield 19 fractions (SA- 1 to SA-19). Fraction-1 (SA1) was re-chromatographed by eluting with $n$-hexane: ethyl acetate (95:5), yielding 5 fractions (SA-1-1 to SA-1-5). One spot was found in SA-1-1 fraction and was named as Compound-1. Moreover, fraction-2 (SA-2) was also separated more through GCC using hexane: ethyl acetate (9:1) eluent to give 6 fractions (SA-2-1 to SA- 
2-6). One spot was discovered form fraction-1 (SA-2-1) and was marked as Compound-2. Hereinafter, Compound- 1 and Compound-2 were elucidated by spectroscopie method in an attempt to determine their stucture.

\section{Result and discussion}

Compound-1 (2mg) was collected as yellow oil. ${ }^{1} \mathrm{H}$ NMR $\delta_{\mathrm{H}}: 1.29$ $(9 \mathrm{H}, \mathrm{s}), 1.34(9 \mathrm{H}, \mathrm{s}), 7.13(1 \mathrm{H}, \mathrm{dd}, J=2.5$ and $8.7 \mathrm{~Hz}), 7.36(1 \mathrm{H}, \mathrm{d}, J=$ $2.5 \mathrm{~Hz}), 7.54(1 \mathrm{H}, \mathrm{d}, J=8.7 \mathrm{~Hz})$. Three protons $\left(\delta_{\mathrm{H}}: 7.13,7.36,7.54\right)$ showed in aromatic protons region. The remaining 18 protons $\left(\delta_{\mathrm{H}}\right.$ : $1.29 \& 1.34)$ supposed to be six methyls. And then, COSY $\left({ }^{1} \mathrm{H}-{ }^{-1} \mathrm{H}\right)$ and $\mathrm{HMBC}$ correlations $\left({ }^{1} \mathrm{H}-{ }^{13} \mathrm{C}\right)$ were joined for confirmation of the structure. The NMR spectra were also compared to previous literature data. ${ }^{14}$ From these data Compound-1 was determined as 2,4-di-tertbutylphenol and the molecular formula was $\mathrm{C}_{14} \mathrm{H}_{22} \mathrm{O}$.
Compound-2 was obtained as pale yellow oil. FTIR spectra showed at 2926-2854 $\mathrm{cm}^{-1}$ ( $\mathrm{sp}^{3} \mathrm{C}-\mathrm{H}$ stretching), $1708 \mathrm{~cm}^{-1}(\mathrm{C}=\mathrm{O}) .{ }^{1} \mathrm{H}$ NMR $\delta_{\mathrm{H}}: 0.77(3 \mathrm{H}, \mathrm{s}), 0.81(3 \mathrm{H}, \mathrm{s}), 0.86(3 \mathrm{H}, \mathrm{s}), 0.89(6 \mathrm{H}, \mathrm{m}), 0.94$ $(3 \mathrm{H}, \mathrm{s}), 0.98(3 \mathrm{H}, \mathrm{s}), 1.19(1 \mathrm{H}, \mathrm{m}), 1.26(24 \mathrm{H}, \mathrm{m}), 1.42(3 \mathrm{H}, \mathrm{m}), 1.50$ $(4 \mathrm{H}, \mathrm{m}), 1.61(3 \mathrm{H}, \mathrm{s}), 1.65(3 \mathrm{H}, \mathrm{m}), 1.67(3 \mathrm{H}, \mathrm{m}), 1.69(3 \mathrm{H}, \mathrm{s}), 1.78($ $1 \mathrm{H}, \mathrm{m}), 1.93(2 \mathrm{H}, \mathrm{m}), 1.98(3 \mathrm{H}, \mathrm{m}), 2.04(2 \mathrm{H}, \mathrm{m}), 2.13(1 \mathrm{H}, \mathrm{m}), 2.23$ $(1 \mathrm{H}, \mathrm{m}), 2.30(2 \mathrm{H}, \mathrm{t}, J=7.5 \mathrm{~Hz}), 4.53(1 \mathrm{H}, \mathrm{dd}, J=3.9,11.5 \mathrm{~Hz}), 5.12$ $(1 \mathrm{H}, \mathrm{t}, J=7.1 \mathrm{~Hz}), 5.25(1 \mathrm{H}, \mathrm{m})$. The proton signal at $\delta_{\mathrm{H}}: 4.53 \mathrm{ppm}(\mathrm{C}-$ 3 ) belonged to the proton attached to the carbon that binds the ester group $(\mathrm{C}-3)$. The proton signal of $5.25(1 \mathrm{H}, \mathrm{m})$ and $5.12(1 \mathrm{H}, \mathrm{t}, J=$ $7.1 \mathrm{~Hz}$ ) linked to $\mathrm{sp}^{2}$ hybridized carbons (C-7 and C-24 respectively). COSY $\left({ }^{1} \mathrm{H}-{ }^{1} \mathrm{H}\right)$ and HMBC correlations $\left({ }^{1} \mathrm{H}-{ }^{13} \mathrm{C}\right)$ were joined for confirmation of structure. NMR spectra also compared to previous literature data. ${ }^{15,16}$ From these data Compound $\mathbf{2}$ was identified as Butyrospermol 3- $\beta$ - $O$-palmitate and molecular formula was $\mathrm{C}_{46} \mathrm{H}_{80} \mathrm{O}_{2}$ (Figure 1) (Figure 2) (Table 1) (Table 2).

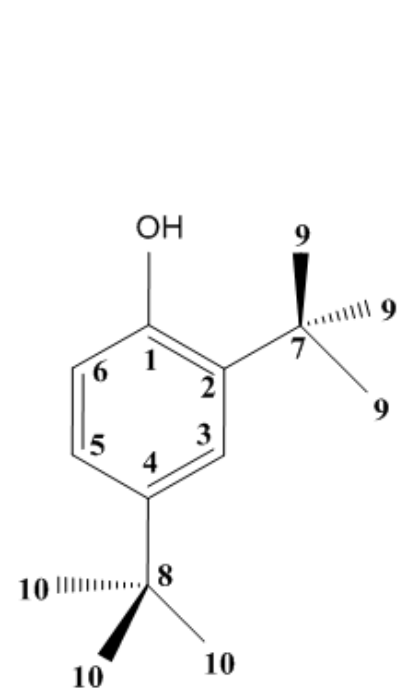

2,4-di-tert-butylphenol

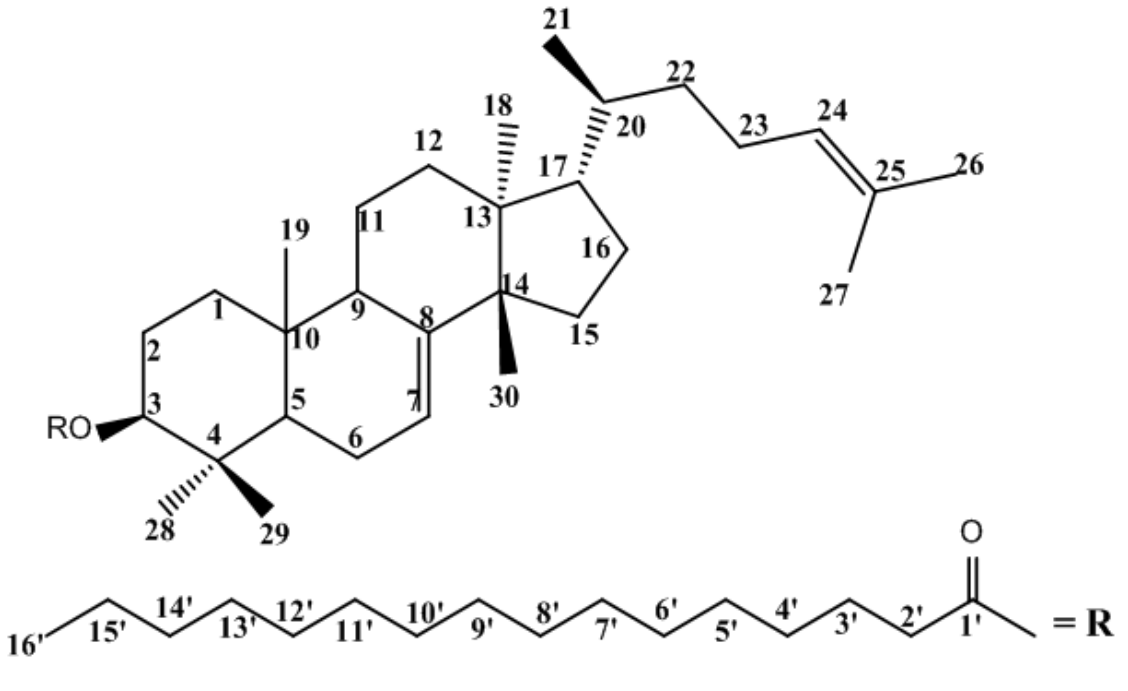

butyrospermol 3-beta- $O$-palmitate

Figure I Chemical structure of 2,4-di-tert-butylphenol and butyrospermol 3- $\beta$-O-palmitate.

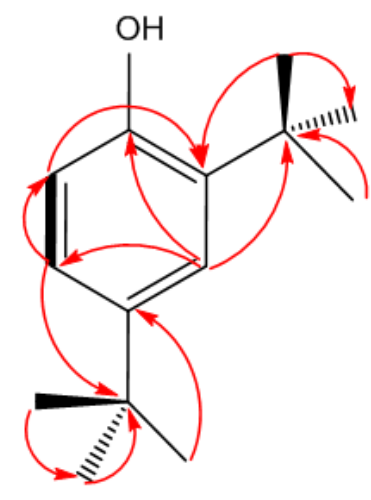

2,4-di-tert-butylphenol

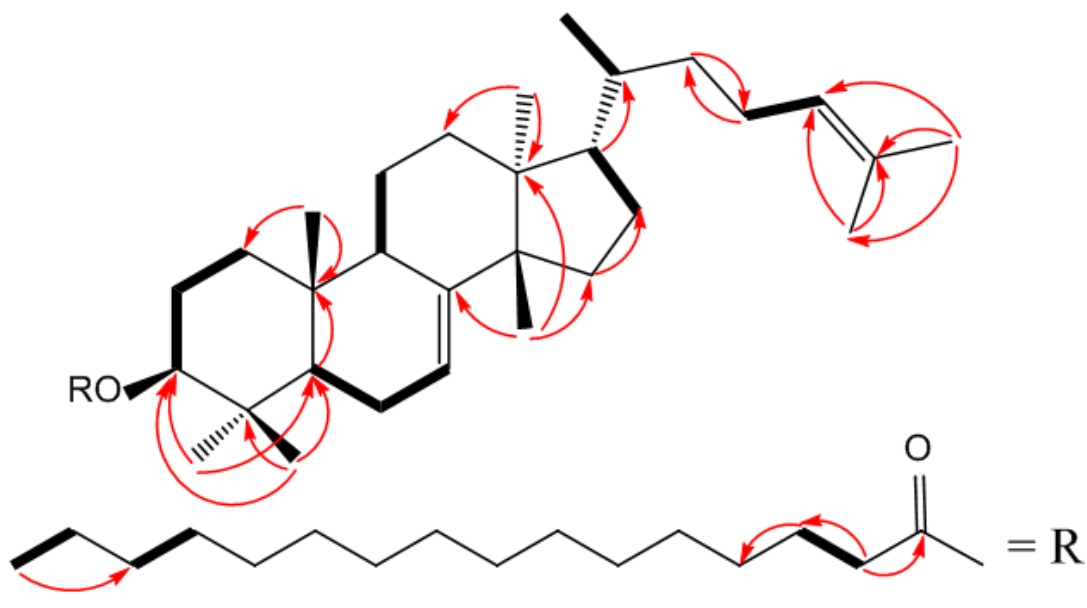

butyrospermol 3-beta- $O$-palmitate

Figure 2 Selected ( ) HMBC, COSY of 2,4-di-tert-butylphenol and butyrospermol 3- $\beta$-O-palmitate. 
Table I NMR data $\left(\mathrm{CDCl}_{3}, 600 \mathrm{MHz}\right)$ of 2,4-di-tert-butylphenol

\begin{tabular}{|c|c|c|c|c|c|}
\hline \multirow{2}{*}{ Position } & \multirow{2}{*}{ DEPT } & \multicolumn{2}{|c|}{ HSQC $\left(' H-{ }^{13} \mathrm{C}\right)$} & \multirow{2}{*}{ HMBC } & \multirow{2}{*}{ cosy } \\
\hline & & $\delta \mathbf{X}$ & $\delta \mathbf{H}$ & & \\
\hline I & C & 147.7 & - & $\mathrm{H}-3$ & - \\
\hline 2 & C & 138.4 & - & $\mathrm{H}-9, \mathrm{H}-6$ & - \\
\hline \multirow[t]{2}{*}{3} & $\mathrm{CH}$ & 124.4 & 7.36 & $\mathrm{H}-5$ & - \\
\hline & & & $(d, J=2.5 \mathrm{~Hz})$ & & \\
\hline 4 & C & 147.1 & - & $\mathrm{H}-6, \mathrm{H}-\mathrm{IO}$ & - \\
\hline \multirow[t]{2}{*}{5} & $\mathrm{CH}$ & 123.9 & 7.13 & $\mathrm{H}-3$ & $\mathrm{H}-6$ \\
\hline & & & $(d d, J=2.5,8.7 \mathrm{~Hz})$ & & \\
\hline \multirow[t]{2}{*}{6} & $\mathrm{CH}$ & 119.1 & 7.54 & $\mathrm{H}-5$ & $\mathrm{H}-5$ \\
\hline & & & $(d, J=8.7 \mathrm{~Hz})$ & & \\
\hline 7 & C & 34.8 & - & $\mathrm{H}-9, \mathrm{H}-3$ & - \\
\hline 8 & C & 34.5 & - & $\mathrm{H}-\mathrm{IO}, \mathrm{H}-5$ & - \\
\hline 9 & $3 \mathrm{CH}_{3}$ & 30.2 & $1.34(\mathrm{~s})$ & - & - \\
\hline 10 & $3 \mathrm{CH}_{3}$ & 31.4 & $1.29(\mathrm{~s})$ & - & - \\
\hline
\end{tabular}

Table $2 \mathrm{NMR}$ data $\left(\mathrm{CDCl}_{3}, 600 \mathrm{MHz}\right)$ of Butyrospermol 3- $\beta$-O-palmitate

\begin{tabular}{|c|c|c|c|c|c|}
\hline \multirow[t]{2}{*}{ Position } & \multirow[t]{2}{*}{ DEPT } & \multicolumn{2}{|c|}{ HSQC ('H- $\left.{ }^{13} \mathrm{C}\right)$} & \multirow[t]{2}{*}{ НМВС } & \multirow[t]{2}{*}{ COSY } \\
\hline & & $13 C$ & $\mathrm{IH}$ & & \\
\hline \multirow[t]{2}{*}{ I } & $\mathrm{CH}_{2}$ & 36.8 & $1.67(\mathrm{~m})$ & $\mathrm{H}-19$ & $\mathrm{H}-2$ \\
\hline & & & $\mathrm{I}, 19(\mathrm{~m})$ & & \\
\hline 2 & $\mathrm{CH}_{2}$ & 25.7 & $\mathrm{I} .67(\mathrm{~m})$ & - & $\mathrm{H}-\mathrm{I}, \mathrm{H}-3$ \\
\hline \multirow[t]{2}{*}{3} & $\mathrm{CH}$ & 81 & 4.53 & $\mathrm{H}-28, \mathrm{H}-29$ & $\mathrm{H}-2$ \\
\hline & & & $(\mathrm{dd}, J=3.9, \mathrm{I} I .5 \mathrm{~Hz})$ & & \\
\hline 4 & C & 38 & - & $\mathrm{H}-29$ & - \\
\hline 5 & $\mathrm{CH}$ & 50.7 & $\mathrm{I} .42(\mathrm{~m})$ & $\mathrm{H}-28, \mathrm{H}-29$ & $\mathrm{H}-6$ \\
\hline \multirow[t]{2}{*}{6} & $\mathrm{CH}_{2}$ & 23.8 & $2.13(\mathrm{~m})$ & - & $\mathrm{H}-5, \mathrm{H}-7$ \\
\hline & & & $\mathrm{I} .98(\mathrm{~m})$ & & \\
\hline 7 & $\mathrm{CH}$ & 117.6 & $5.25(\mathrm{~m})$ & - & $\mathrm{H}-6$ \\
\hline 8 & C & 146 & - & $\mathrm{H}-30$ & - \\
\hline 9 & $\mathrm{CH}$ & 48.8 & $2.23(\mathrm{~m})$ & - & $\mathrm{H}-\mathrm{II}$ \\
\hline 10 & C & 34.8 & - & $\mathrm{H}-19, \mathrm{H}-5$ & - \\
\hline II & $\mathrm{CH}_{2}$ & 18.2 & $\mathrm{I} .50(\mathrm{~m})$ & - & $\mathrm{H}-9$ \\
\hline \multirow[t]{2}{*}{12} & $\mathrm{CH}_{2}$ & 33.8 & $\mathrm{I} .65(\mathrm{~m})$ & $\mathrm{H}-\mathrm{I} 8$ & - \\
\hline & & & $\mathrm{I} .78(\mathrm{~m})$ & & \\
\hline 13 & C & 43.6 & - & $\mathrm{H}-\mathrm{I} 8, \mathrm{H}-30$ & - \\
\hline 14 & C & 51.9 & - & $\mathrm{H}-18, \mathrm{H}-30$ & - \\
\hline \multirow[t]{2}{*}{15} & $\mathrm{CH}_{2}$ & 34 & $\mathrm{I} .42(\mathrm{~m})$ & $\mathrm{H}-30$ & - \\
\hline & & & $\mathrm{I} .50(\mathrm{~m})$ & & \\
\hline 16 & $\mathrm{CH}_{2}$ & 29.2 & $\mathrm{I} .26(\mathrm{~m})$ & $\mathrm{H}-\mathrm{I} 5$ & $\mathrm{H}-\mathrm{I} 7$ \\
\hline
\end{tabular}




\begin{tabular}{|c|c|c|c|c|c|}
\hline \multirow{2}{*}{$\begin{array}{l}\text { Position } \\
17\end{array}$} & \multirow{2}{*}{$\begin{array}{l}\text { DEPT } \\
\mathrm{CH}\end{array}$} & \multicolumn{2}{|c|}{ HSQC $\left({ }^{\prime} H-{ }^{13} \mathrm{C}\right)$} & \multirow{2}{*}{ HMBC } & \multirow{2}{*}{$\begin{array}{l}\text { cosy } \\
\mathrm{H}-16\end{array}$} \\
\hline & & 53.2 & $1.50(\mathrm{~m})$ & & \\
\hline 18 & $\mathrm{CH}_{3}$ & 22.1 & $0.81(\mathrm{~s})$ & - & - \\
\hline 19 & $\mathrm{CH}_{3}$ & 13.1 & $0.77(\mathrm{~s})$ & - & - \\
\hline 20 & $\mathrm{CH}$ & 35.7 & $1.42(\mathrm{~m})$ & $\mathrm{H}-17$ & $\mathrm{H}-2 \mathrm{I}$ \\
\hline 21 & $\mathrm{CH}_{3}$ & 18.2 & $0.89(\mathrm{~m})$ & - & $\mathrm{H}-20$ \\
\hline 22 & $\mathrm{CH}_{2}$ & 39.8 & $\mathrm{I} .98(\mathrm{~m})$ & $\mathrm{H}-23$ & - \\
\hline 23 & $\mathrm{CH}_{2}$ & 26.8 & $2.04(\mathrm{~m})$ & $\mathrm{H}-22$ & $\mathrm{H}-24$ \\
\hline \multirow[t]{2}{*}{24} & $\mathrm{CH}$ & 125.1 & 5.12 & $\mathrm{H}-26, \mathrm{H}-27$ & $\mathrm{H}-23$ \\
\hline & & & $(\mathrm{t}, \mathrm{J}=7.1 \mathrm{~Hz})$ & & \\
\hline 25 & C & 130.9 & - & $\mathrm{H}-26, \mathrm{H}-27$ & - \\
\hline 26 & $\mathrm{CH}_{3}$ & 17.7 & $1.61(\mathrm{~s})$ & $\mathrm{H}-27$ & - \\
\hline 27 & $\mathrm{CH}_{3}$ & 25.7 & $1.69(\mathrm{~s})$ & $\mathrm{H}-26$ & - \\
\hline 28 & $\mathrm{CH}_{3}$ & 27.7 & $0.86(s)$ & - & - \\
\hline 29 & $\mathrm{CH}_{3}$ & 16 & $0.94(s)$ & - & - \\
\hline 30 & $\mathrm{CH}_{3}$ & 27.3 & $0.98(s)$ & $\mathrm{H}-15$ & - \\
\hline I' & C & 173.6 & - & $\mathrm{H}-2^{\prime}$ & - \\
\hline \multirow[t]{2}{*}{$2^{\prime}$} & $\mathrm{CH}_{2}$ & 35 & 2.3 & - & $\mathrm{H}-3^{\prime}$ \\
\hline & & & $(\mathrm{t}, \mathrm{J}=7.5 \mathrm{~Hz})$ & & \\
\hline $3^{\prime}$ & $\mathrm{CH}_{2}$ & 25.1 & $\mathrm{I} .65(\mathrm{~m})$ & $\mathrm{H}-2^{\prime}$ & $\mathrm{H}-2^{\prime}$ \\
\hline $4^{\prime}$ & $\mathrm{CH}_{2}$ & 29.7 & $1.26(\mathrm{~m})$ & H-3' & \\
\hline 5 & $\mathrm{CH}_{2}$ & 29.7 & $1.26(\mathrm{~m})$ & - & - \\
\hline $6^{\prime}$ & $\mathrm{CH}_{2}$ & 29.6 & $1.26(\mathrm{~m})$ & - & - \\
\hline 7 & $\mathrm{CH}_{2}$ & 29.6 & $1.26(\mathrm{~m})$ & - & - \\
\hline 8 & $\mathrm{CH}_{2}$ & 29.6 & $1.26(\mathrm{~m})$ & - & - \\
\hline $9^{\prime}$ & $\mathrm{CH}_{2}$ & 29.5 & $1.26(\mathrm{~m})$ & - & - \\
\hline 10 & $\mathrm{CH}_{2}$ & 29.4 & $\mathrm{I} .26(\mathrm{~m})$ & - & - \\
\hline II' & $\mathrm{CH}_{2}$ & 29.3 & $1.26(\mathrm{~m})$ & - & - \\
\hline 12 & $\mathrm{CH}_{2}$ & 29.2 & $1.26(\mathrm{~m})$ & - & - \\
\hline $13^{\prime}$ & $\mathrm{CH}_{2}$ & 28.4 & $1.93(\mathrm{~m})$ & - & $\mathrm{H}-14$ \\
\hline 14 & $\mathrm{CH}_{2}$ & 31.9 & $1.26(\mathrm{~m})$ & $\mathrm{H}-16^{\prime}$ & $\mathrm{H}-13^{\prime}$ \\
\hline $15^{\prime}$ & $\mathrm{CH}_{2}$ & 22.7 & $\mathrm{I} .26(\mathrm{~m})$ & - & $\mathrm{H}-16^{\prime}$ \\
\hline 16 & $\mathrm{CH}_{3}$ & 14.1 & $0.89(\mathrm{~m})$ & - & $\mathrm{H}-15$ \\
\hline
\end{tabular}

The bioactivities of 2,4-Di-tert-butylphenol has been previously reported such as moderate cytotoxicity against HeLa and MCF-7, high percentage of antioxidant activity, active antibacterial against Pseudomonas aeruginosa and Staphylococcus aureus, prevention the fungal mycelial growth of Aspergillus niger, Fusarium oxysporum and Penicillium chrysogenumon. ${ }^{17-19}$ However, Butyrospermol 3- $\beta-O-$ palmitate hasn't been reported any bioactivities in literature. Therefore, we would like to recommend studying the various bioactivities of this compound for the next research. 


\section{Conclusion}

In this study, 2,4-di-tert-butylphenol and Butyrospermol 3- $\beta-O-$ palmitate were isolated from stem bark of $S$. aqueum. These phytochemical constituents are the first to be reported from the stem bark of this species.

\section{Acknowledgments}

The authors would like to special thanks Faculty of Science and Technology, Universitas Airlangga and Faculty of Pharmacy, Meijo University, Tempaku, Nagoya, Japan.

\section{Funding}

Universitas Airlangga supported the first author for the scholarship (ADS) and The Indonesian Ministry of Research, Technology, and Higher Education for research grant (Basic Research, 2020).

\section{Conflicts of interest}

The authors declare there are no conflicts of interest.

\section{References}

1. Insanu M, Ramadhania ZM, Halim EN, et al. Isolation of 5,7-Dihydroxy, 6,8-Dimethyl Flavanone from Syzygium aqueum with its antioxidant and xanthine oxidase inhibitor activities. Pharmacognosy Research. 2018;10:24-30.

2. Vasconcelos TNNC, Proença CEB, Ahma B, et al. Myrteae phylogeny, calibration, biogeography and diversification patterns: Increased understanding in the most species rich tribe of Myrtaceae. Molecular Phylogenetics and Evolution. 2017;109:113-137.

3. Manaharana T, Chakravarthi S, Radhakrishnan AK, et al. In vivo toxicity evaluation of a standardized extract of Syzygium aqueum leaf. Toxicology Reports. 2014;1:718-725.

4. Sagala E, Sihombing H, Agustini F, et al. Analysis of consumer demand on Syzygium aqueum In North Sumatera, Indonesia. IOSR Journal of Economics and Finance. 2017;5(1):44-48.

5. Palanisamy UD, Ling LT, Manaharan T, et al. Standardized extract of Syzygium aqueum: a safe cosmetic ingredient. International Journal of Cosmetic Science. 2011;33:269-275.

6. Manaharan T, Appleton D, Cheng HM, et al. Flavonoids isolated from Syzygium aqueum leaf extract as potential antihyperglycaemic agents. Food Chemistry. 2012;132:1802-1807.

7. Osman H, Rahim AA, Isa NM, et al. Antioxidant activity and phenolic content of paederia foetida and Syzygium aqueum. Molecules. 2009:970 978.
8. Saptarini NM, Herawat IE. Antioxidant activity of water apple (Syzygium aqueum) fruit and fragrant mango (Mangifera odorata) fruit. Asian Journal of Pharmaceutical and Clinical Research. 2017:54-56.

9. Lim ASL, Rabeta MS. Proximate analysis, mineral content and antioxidant capacity of milk apple, malay apple and water apple. International Food Research Journal. 2013;20(2):673-679.

10. Rocchet G, Lucini L, Ahmed SR, et al. In vitro cytotoxic activity of six Syzygium leaf extracts as related to their phenolic profiles: An untargeted UHPLC-QTOF-MS approach. Food Research International. 2019;126:123.

11. Sobeh M, Mona F, Mahmoud MF, et al. Syzygium aqueum: A polyphenolrich leaf extract exhibits antioxidant, hepatoprotective, pain-killing and anti-inflammatory activities in animal models. Forntiers in Pharmacology. 2018;9(6):1-14.

12. Nonaka G, Aiko Y, Aritake $\mathrm{K}$, et al. Samarangenins A and B, Novel proanthocyanidins with doubly bonded structures, form Syzygium samarangense and S. aqueum. Chemical and Pharmaceutical Bulletin. 1992; 40(10):2671-2673.

13. Tukiran, Mahmudah F, Hidayati N, Shimizu K. A phenolic acid and its antioxidant activity from stem bark of chloroform fraction of Syzygium littorale (blume) amshoff (myrtaceae). Molekul. 2016;11(2):180-189.

14. Chawawisit K, Bhoopong P, Phupong W, et al. 2, 4-Di-tert-butylphenol, the bioactive compound produced by Streptomyces sp. KB1. Journal of Applied Pharmaceutical Science. 2015;5(3);7-12.

15. Wua J, Lia M, Xiao Z, Zhou Y. Butyrospermol fatty acid esters from the fruit of a chinese mangrove Xylocarpus granatum. Verlag der Zeitschrift fü Naturforschung. 2006;61(11):1447-1449.

16. Pietro MED, Mannu A, Mele A. NMR determination of free fatty acids in vegetable oils. Processes. 2020;8(410):1-15.

17. Zhao F, Wang P, Lucardi RD, et al. Natural sources and bioactivities of 2,4-Di-Tert-Butylphenol and its analogs fuqiang. Toxins. 2020;12(35):126.

18. Varsha KK, Devendra L, Shilp G, et al. 2,4-Di-tert-butyl phenol as the antifungal, antioxidant bioactive purified from a newly isolated Lactococcus sp. International Journal of Food Microbiology. 2015;211:44-50.

19. Aissaoui N, Mahjoubi M, Nas F, et al. Antibacterial potential of 2,4-Ditert-butylphenol and calixarene-based prodrugs from thermophilic Bacillus licheniformis isolated in algerian hot spring. Geomicrobiology Journal. 2018:1-10. 